\title{
2 cases of Pneumocystis Jiroveci Pneumonia during treatment of Cushing's Syndrome. Is there a case for prophylaxis of PJP in the treatment of severe hypercortisolism?
}

Hunter A, McCance DR, Hunter SJ, Walsh J

Regional Centre for Endocrinology and Diabetes, Royal Victoria Hospital, Belfast, Northern Ireland

\section{Introduction}

Pneumocystis Jiroveci Pneumonia (PJP) is well recognised in HIV infected and transplant recipient populations and prophylaxis is standard practice. However PJP may also occur in rarer cases of immunodeficiency. We report 2 cases of Cushing's syndrome complicated by PJP.

\section{Case 1: Cushing's Disease}

A 30 year old man with no past medical history presented with bloody diarrhoea, abdominal pain, lethargy, facial swelling, weight gain, shortness of breath, leg weakness and mood disturbance. On examination he was cushingoid with fresh striae, thin bruised skin, proximal myopathy and bilateral pitting oedema. There was bilateral knee swelling with moderate effusions and oral candidiasis. Visual fields were intact to confrontation. He was hypertensive and hyperglycaemic $(20.1 \mathrm{mmol} / \mathrm{mol})$. He was admitted to the regional endocrinology unit for investigation.

During his admission the patient developed a severe hospital acquired pneumonia which failed to respond to Tazocin. This was felt to be due to his immunocompromised state and he was commenced on Metyrapone. 48 hours later he deteriorated and required intubation, ventilation and dialysis in intensive care. An Etomidate infusion was started to maintain an early morning cortisol of 500-1000 nmol/L. Laboratory results were positive for Pneumocystis Jiroveci, Tuberculosis, Strep. Pneumoniae, Influenza A (H1 swine lineage), cytomegalovirus, RSV and Candida Albicans.

\begin{tabular}{l|l|l}
\multicolumn{1}{c|}{ Investigation } & Result & Reference Range \\
\hline Morning Cortisol & $1894 \mathrm{nmol} / \mathrm{L}$ & $166-507 \mathrm{nmol} / \mathrm{L}$ \\
ACTH & $262 \mathrm{ng} / \mathrm{L}$ & $<46 \mathrm{ng} / \mathrm{L}$ \\
Urinary Cortisol & $>266,786 \mathrm{nmol} / 24 \mathrm{~h}$ & $<210 \mathrm{nmol} / 24 \mathrm{~h}$
\end{tabular}

Dexamethasone suppression No suppression of cortisol with low or high dose $\mathrm{CRH}$ stimulation

MRI Pituitary
No significant increase in cortisol or ACTH $15 \mathrm{~mm}$ macroadenoma

Advice was sought from Infectious Diseases and he was treated with cotrimoxazole, quadruple TB therapy and ganciclovir. Progress was slow and after a life threatening illness in ICU he was discharged to the ward for metyrapone titration and 1 week of further haemodialysis. The risk of further infection was considered too great to stop metyrapone for petrosal sinus sampling. Following a CT chest, abdomen and pelvis and an octreotide scan to exclude an ectopic source of ACTH, it was concluded that the diagnosis was that of Cushing's Disease. At trans sphenoidal surgery, an ACTH positive pituitary adenoma was resected and following radiotherapy he is now in remission and no longer requires PJP prophylaxis.

\section{Case 2: Cushing's Syndrome from Adrenal Cancer}

A 59 year old male presented with a 2-3 week history of shortness of breath and peripheral oedema. He had been diagnosed with diabetes 2 months prior to this and had a 3 year history of hypertension. On examination there was bilateral oedema to his knees, ascites, bruised thin skin, proximal myopathy, central obesity and cushingoid facies. Further history revealed a 6 month history of proximal muscle weakness, abdominal distention and weight gain. CT imaging showed a $17 \times 12.5 \times 15.4 \mathrm{~cm}$ mass arising from the left adrenal gland (figure 1) along with pulmonary and hepatic metastases. A diagnosis of PT4N1M1 adrenal adenocarcinoma was made. Cushing's Syndrome was confirmed when cortisol failed to suppress with $1 \mathrm{mg}$ dexamethasone at $906 \mathrm{nmol} / \mathrm{L}$ and urinary cortisol was elevated at $1357 \mathrm{nmol} / 24 \mathrm{~h}(<210 \mathrm{nmol} / 24 \mathrm{~h})$.

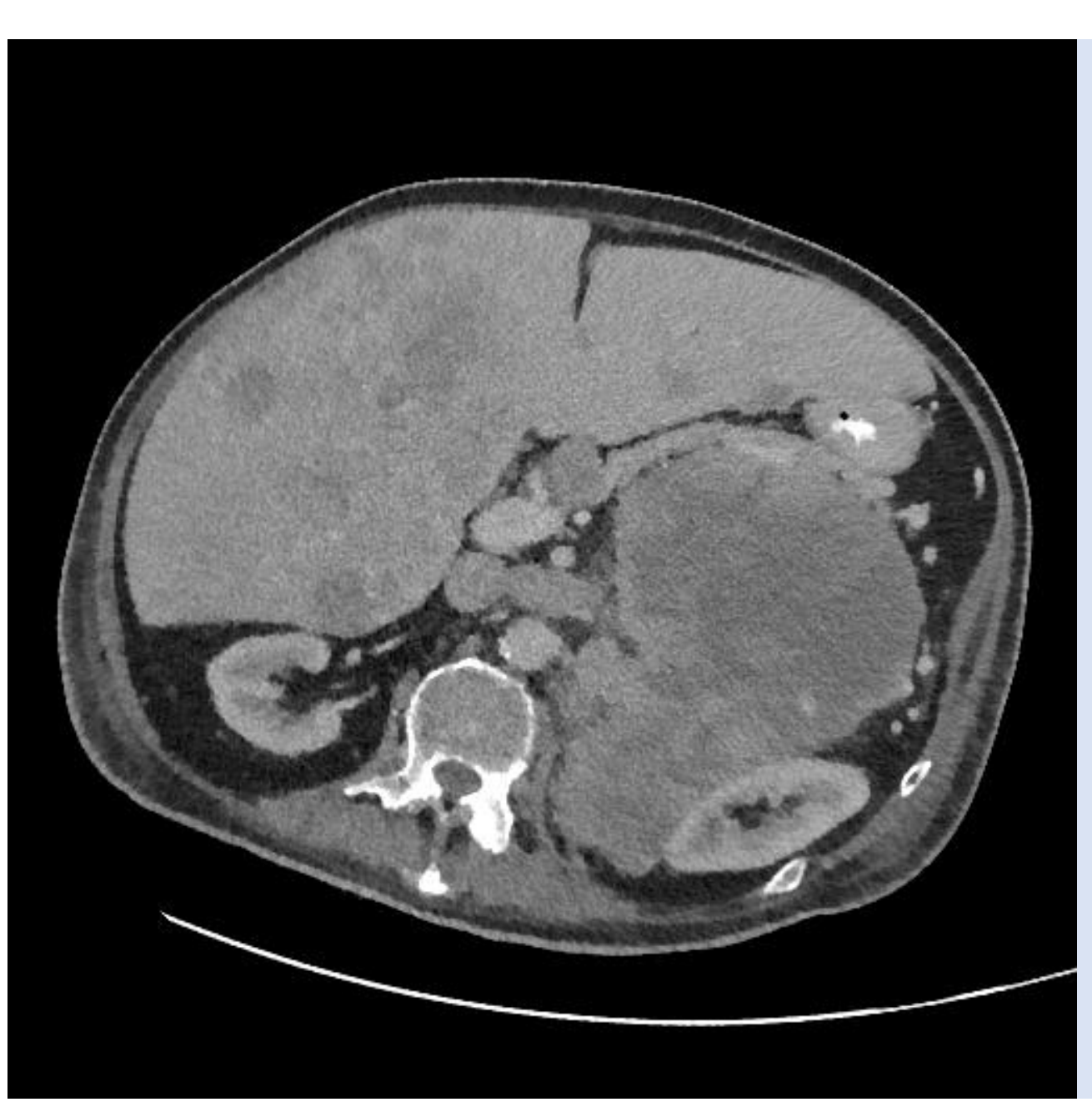

Figure 1

Computerised Tomography image of the abdomen showing lesion arising from left adrenal gland

Following review with Oncology and Endocrinology a titrating regimen of Mitotane was commenced. Due to the severity of hypercortisolism hydrocortisone replacement was delayed. 10 days after starting mitotane he was admitted with cough and shortness of breath. He was initially treated as community acquired pneumonia however Cotrimoxazole was also prescribed. He deteriorated early in the admission and required $80 \%$ oxygen for type one respiratory failure. Antibiotics were escalated and metyrapone was added because morning cortisol remained elevated at $1200 \mathrm{nmol} / \mathrm{L}$ on Mitotane. Cortisol fell to $527 \mathrm{nmol} / \mathrm{L}$ and he was commenced on dexamethasone $0.5 \mathrm{mg}$. Swabs taken for respiratory pathogens were positive for Pneumocystis Jiroveci. There was no bacterial growth on sputum culture and virology was negative. The patient developed a drug rash to cotrimoxazole and therefore was switched to clindamycin and primaquine for active treatment of PJP on the advice of Infectious Diseases.

He received 21 days of treatment for PJP and was then placed on dapsone for prophylaxis. Following discharge from hospital he had 2 cycles of chemotherapy but continued to deteriorate and died expectedly at home 5 months from diagnosis.

These 2 cases of severe hypercortisolism were complicated by PJP infection following initiation of cortisol lowering therapy. PJP is known to be associated with severe hypercortisolism ${ }^{1}$ which is defined by the Endocrine Society as urinary free cortisol over 5 times the normal limit ${ }^{2}$. In our laboratory this would be a urinary cortisol greater than $1050 \mathrm{nmol} / 24 \mathrm{~h}$. It is thought that patients with severe hypercortisolism carry Pneumocystis Jiroveci in their lungs. When cortisol is lowered immune reconstitution occurs and the anti-inflammatory effect of excess steroid is lost resulting in pneumocystis pneumonia ${ }^{1,3}$. The mortality of PJP in Cushing's patients is estimated at $60-65 \%^{1}$. Currently prophylaxis of PJP is not routinely incorporated into the treatment of Cushing's. We propose that PJP prophylaxis should be carefully considered in patients with severe hypercortisolism and call for clearer guidance.

References

1. Van Halem K1, Vrolijk L1, Pereira AM2, de Boer MGJ1. Characteristics and Mortality of Pneumocystis Pneumonia in Patients With Cushing's Syndrome: A Plea for Timely Initiation of Chemoprophylaxis. Open Forum Infect Dis. 2017 Jan 30;4(1):ofx002.

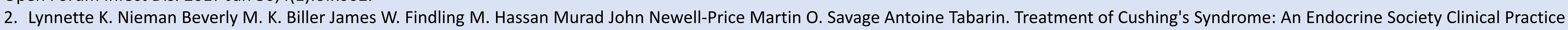
Guideline. The Journal of Clinical Endocrinology \& Metabolism, Volume 100, Issue 8, 1 August 2015, Pages 2807-2831.

3. Sarlis NJ1, Chanock SJ, Nieman LK. Cortisolemic indices predict severe infections in Cushing syndrome due to ectopic production of adrenocorticotropin. J Clin Endocrinol Metab. 2000 Jan;85(1):42-7. 